\title{
OPEN Evaluation of antibiotic-induced taste and smell disorders using the FDA adverse event reporting system database
}

\begin{abstract}
Yusuke Kan ${ }^{1,2}$, Junko Nagai ${ }^{1} \&$ Yoshihiro Uesawa ${ }^{1 凶}$
Adverse effects can occur owing to anorexia, which can reduce treatment compliance and worsen the patients overall condition. One such side effect, namely drug-induced taste and smell disorders, reduces patients quality of life. Although antibiotics can cause taste and smell disorders, a few studies have examined antibiotic-induced taste and smell disorders. Therefore, this study comprehensively analyzed the relationship between taste and smell disorders and antibiotic usage. The side effects of antibiotics were investigated using the FDA Adverse Event Reporting System database (FAERS). The reporting odds ratios between the listed drugs and taste and smell disorders $P$ values were comprehensively calculated. Adjusted odds ratios were calculated to account for patient background. Furthermore, to clarify the feature of this adverse effect, shape parameters indicating the expression pattern were calculated. Signals that induced taste and smell disorders were detected for six antibiotics, including drugs for which this event is not described in the package insert in Japan. Multiple logistic regression analysis suggested an association of taste and smell disorders with gender, hypertension, mental disorder, and cancer. The median time to onset of antibiotic-induced taste and smell disorders was 2-5 days. Six antibiotics could be analyzed, and four of these drugs matched those with detected signals. Our study supported previous findings on gender and age. Furthermore, antibiotic-induced taste and smell disorders are likely to develop in the early stage of treatment. For these reasons, it is important to remember the risk of developing of taste and smell disorders when administering antibiotics. In addition, it is recommended that the patient be monitored carefully for at least 1 week before initiating treatment, and the patients course should be followed for at least 2 months.
\end{abstract}

Although taste and smell disorders are not a life-threatening adverse event (AE), it is believed to reduce patients' quality of life and decrease treatment compliance. Furthermore, in severe cases, anorexia can affect the patients general condition. In the American National Health and Nutrition Examination Survey 2011-2012 involving 140 million adult respondents, more than 5\% experienced taste and smell disorders, and it has been reported that $10 \%$ of patients have experienced the olfactory disorder. Patients with smell disorders are often aware of taste disorders; however, several patients confuse taste disorder with smell disorder, which is a taste disorder. Furthermore, chronic kidney disease, heart disease, malignant tumors, psychiatric disorders, and depression are chronic diseases associated with taste and smell disorders ${ }^{1-3}$. To date, $50 \%$ of patients using levofloxacin inhalation therapy have been reported to possibly have taste and smell disorders. In another study, dysgeusia was reported in patients receiving a combination of ciprofloxacin and tinidazole ${ }^{4}$.

Drug-induced taste disorder in Japan accounts for about $16 \%$ of outpatient cases, and smell disorder account for approximately $3 \%$. It has also been reported that standard zinc replacement therapy requires a longer period of treatment than other causes ${ }^{5,6}$. In Japan, the incidence of predominant dysgeusia peaked in patients in their $50 \mathrm{~s}$ and $60 \mathrm{~s}$ in the 1980s, but it has recently been reported that the onset is more frequent after the $60 \mathrm{~s}$ decade of life ${ }^{7}$.

Chemotherapeutic agents, other anticancer drugs, ACE inhibitors, and antibiotics have been reported to induce dysgeusia ${ }^{6,7}$. Whereas the drugs are used only for specific patient groups, antibiotics may be used in most patients. Analysis of the time to onset of taste and smell disorders during macrolide and fluoroquinolone therapy has shown that most taste and smell disorders developed within one month of taking the antibiotics. In addition,

${ }^{1}$ Department of Medical Molecular Informatics, Meiji Pharmaceutical University, Tokyo 204-8588, Japan. ${ }^{2}$ Nanohana Pharmacy, Tokyo 196-0014, Japan. ${ }^{\circledR}$ email: vesawa@my-pharm.ac.jp 


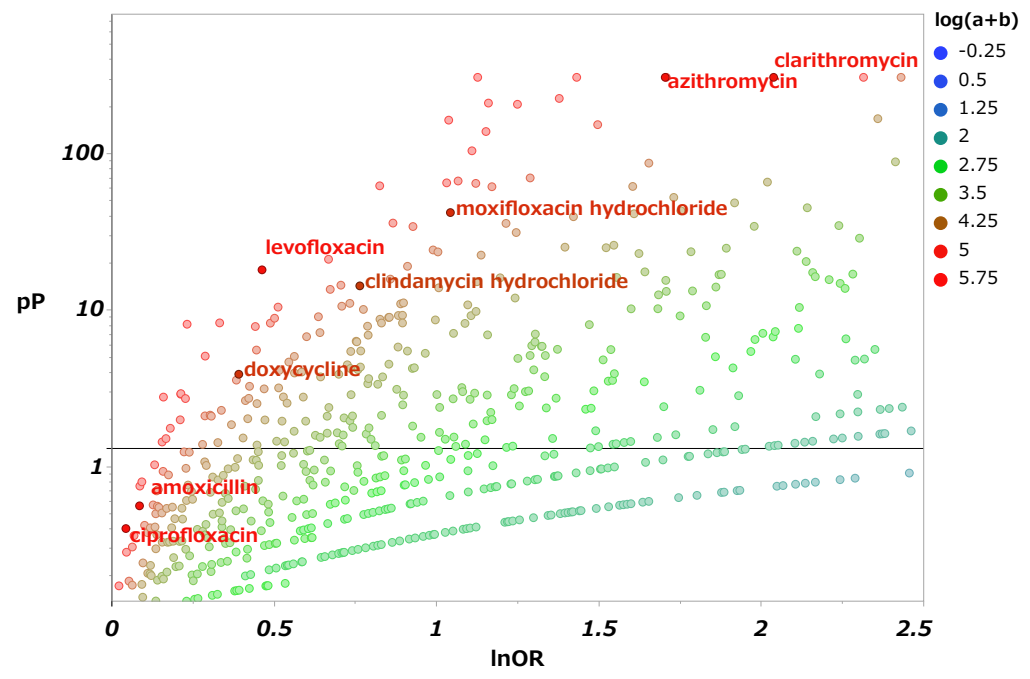

Figure 1. Taste and smell disorders associated with pharmaceuticals. This figure presents the relationships between taste and smell disorders and suspected causative drugs. The X-axis is the logarithm of the reporting odds ratio (lnOR), and the $\mathrm{Y}$-axis is the negative logarithm of the $P$ value calculated using Fisher's exact test $(-\log P$ value; $\mathrm{pP})$. The horizontal line in the figure indicates the reference line at $\mathrm{pP}=1.3(P=0.05) . \mathrm{a}+\mathrm{b}$ indicates the number of reports of taste and smell disorders, and increasing feature is represented by a change in color from blue to red.

they report that in severe cases of taste and smell disorders caused by macrolides and fluoroquinolones, there can be a permanent taste and smell disorders. When attributed to macrolides, $58 \%$ took more than a week to disappear. However, it was reported that in $7 \%$ of cases, taste and smell disorders did not disappear after 150 days of taking the drug ${ }^{8}$. The study by Marco et al. (2011) provided an analysis of such AEs by class of antibiotic. However, no detailed analysis of each drug was reported. In this study, by clarifying the relationship between taste and smell disorders and each antibiotic, we aim to obtain information that contributes to reducing AEs. We used the FDA Adverse Event Reporting System (FAERS) to assess AEs based on the reporting odds ratio (ROR) ${ }^{9-13}$. We examined the relationship between antibiotics and taste and smell disorders by examining the number of reports. In addition, we examined the covariates such as patient background using adjusted odds ratios (AdORs) via multiple logistic regression analysis ${ }^{14,15}$. In addition, using time information data, an analysis of AE onset time using weibull distribution was performed to examine the AE onset time profile for each drug ${ }^{16-18}$. This is the first report to comprehensively analyze the relationship between antibiotics and taste and smell disorders.

\section{Results}

A scatter plot of the relationship between the ROR and statistically significant differences, a volcano plot, was made to examine antibiotics that caused taste and smell disorders (Fig. 1). In this figure, the drugs in the upper right of the scatter plot indicate more likely to cause taste and smell disorders.

The total number of reports in the FAERS database was 43,955,583, including 78,974 reports of taste and smell disorders. The lower limit of the $95 \%$ confidence interval (CI) of ROR for these antibiotics exceeded 1 , indicating that a signal was detected (Table 1). Multiple components of these antibiotics have been reported (see Supplementary Table S1).

Since it was confirmed that similar results could be obtained when the total number of reports was added up, the maximum reported component of each antibiotic was used as the representative value in this study (see Supplementary Table S2).

Furthermore, to comprehensively examine the feature of taste and smell disorders by gender, volcano plots were created to show the relationship between ROR and the $P$ value for each AEs. The results illustrated that antibiotic-induced taste and smell disorders was more common in women than in men (Fig. 2, Table 2). Next, we created a volcano plot for older and younger individuals' difference, and comprehensively investigated the tendency of taste and smell disorders with age. The results demonstrated that older individuals, defined as an age of at least 60 years, were more likely to develop taste and smell disorders than younger individuals. When defined at the ages of 65 and 70, no significant results were obtained, and the number of reports was biased. Based on these results, the older group comprised people aged 60 years and older (Fig. 2, Table 2). Comparing the number of reports in each age group, taste and smell disorders was frequent among patients in their $50 \mathrm{~s} \mathrm{to} 70 \mathrm{~s}$ (Fig. 3).

Concerning the time to onset of taste and smell disorders, the median duration of antibiotic therapy was 1 week (inter quartile range: $1-6$ days). Because the calculated $\beta$ was less than 1 and the $95 \%$ CI did not include 1 , the onset of taste and smell disorders was found to decrease over time. (Fig. 4, Table 3).

AdORs were calculated after controlling for covariates using multiple logistic regression analysis. As a result, AdOR for indicated diseases of hypertension, psychiatric disorders and cancer was greater than 1.0 and above 


\begin{tabular}{|l|l|l|l|l|l|l|l|}
\hline Drug & ROR & 95\%CI & P value & a & b & c & d \\
\hline Amoxicillin & 1.09 & $(0.94-1.26)$ & 0.2744 & 179 & 91,597 & 78,795 & $43,785,012$ \\
\hline Azithromycin & 5.5 & $(5.15-5.87)$ & $<.0001$ & 911 & 93,005 & 78,063 & $43,783,604$ \\
\hline Ciprofloxacin & 1.04 & $(0.95-1.15)$ & 0.3962 & 397 & 211,546 & 78,577 & $43,665,063$ \\
\hline Clarithromycin & 7.67 & $(7.22-8.16)$ & $<.0001$ & 1045 & 76,575 & 77,929 & $43,800,034$ \\
\hline Clindamycin & 2.15 & $(1.81-2.54)$ & $<.0001$ & 134 & 34,853 & 78,840 & $43,841,756$ \\
\hline Doxycycline & 1.48 & $(1.23-1.78)$ & 0.0001 & 110 & 41,549 & 78,864 & $43,835,060$ \\
\hline Levofloxacin & 1.59 & $(1.44-1.75)$ & $<.0001$ & 429 & 150,575 & 78,545 & $43,726,034$ \\
\hline Moxifloxacin & 2.84 & $(2.50-3.22)$ & $<.0001$ & 239 & 47,007 & 78,735 & $43,829,602$ \\
\hline
\end{tabular}

Table 1. ROR of antibiotics that cause taste and smell disorders. a Number of reports of taste and smell disorders due to antibiotics, b: Number of reports of taste and smell disorders other than antibiotics, c: Number of reports of other AEs due to antibiotics, d: Number of reports of other AEs other than antibiotics. The results of the analysis of clindamycin and moxifloxacin indicate those of clindamycin hydrochloride and moxifloxacin hydrochloride, respectively. (see Supplementary Table S1-S2.)
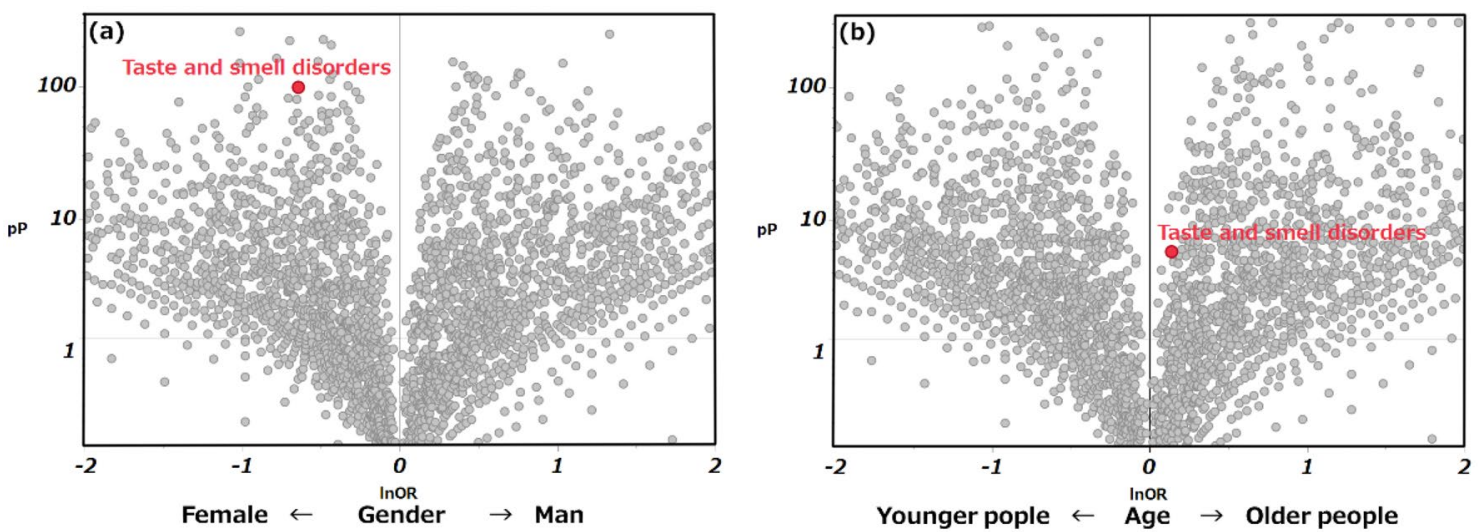

Figure 2. Relationship between antibiotic-induced taste and smell disorders and gender/age. Volcano plots of taste and smell disorders and gender differences/age due to antibiotics. (a) It was suggested that this was more likely to occur in women than in men. (b) Older people are defined as being over 60 years old. It was suggested that it is more likely to occur in older people over 60 years old than in younger people.

\begin{tabular}{|l|l|l|l|l|l|l|l|l|}
\hline & $\%$ & ROR & 95\%CI & $P$ value & a & b & c & d \\
\hline Gender (man) & 0.5 & 0.5 & $(0.49-0.56)$ & $<.0001$ & 1493 & 3194 & 949,502 & 1067421 \\
\hline Older people $(\geqq 60)$ & 0.5 & 1.2 & $(1.09-1.22)$ & $<.0001$ & 2293 & 2394 & 915,797 & 1101126 \\
\hline Older people $(\geqq 65)$ & 0.4 & 1 & $(0.95-1.07)$ & 0.762 & 1726 & 2961 & 738,281 & 1278642 \\
\hline Older people $(\geqq 70)$ & 0.3 & 0.9 & $(0.88-1.00)$ & 0.057 & 1216 & 3471 & 548,159 & 1468764 \\
\hline
\end{tabular}

Table 2. Relationships of antibiotic-induced taste and smell disorders with gender and age. a: Number of cases of taste and smell disorders in older males. b: Number of cases of taste and smell disorders in younger females. c: Number of other AEs in older males. d: Number of cases of other AEs in younger females.

the lower limit of the $95 \%$ CI concomitant disease. In addition, the antibiotic AdORs were greater than 1.0 and above the lower limit of the $95 \%$ CI for azithromycin, clarithromycin, doxycycline, levofloxacin, and moxifloxacin (Table 4). The $P$ value of the estimated model was significant at $P<0.0001$. The fit of the model was confirmed by the goodness of fit test and the estimated model was appropriate.

Spearman's squared rank correlation coefficient $(\rho)$ was less than 0.9 for all combinations. For this reason, no internal correlation was observed among the items used in this analysis, and thus, they were treated as independent factors.

The need to evaluate the possibility of false signals owing to confounding factors in the analysis of adverse drug reaction databases in FAERS has been highlighted ${ }^{19}$. We calculated signal intensity adjusted for confounding factors to account for the possibility of detecting a false signal owing to such confounding factors (Table 5). We concluded that clindamycin was a false signal. A decrease in signal intensity was observed for three antibiotics 


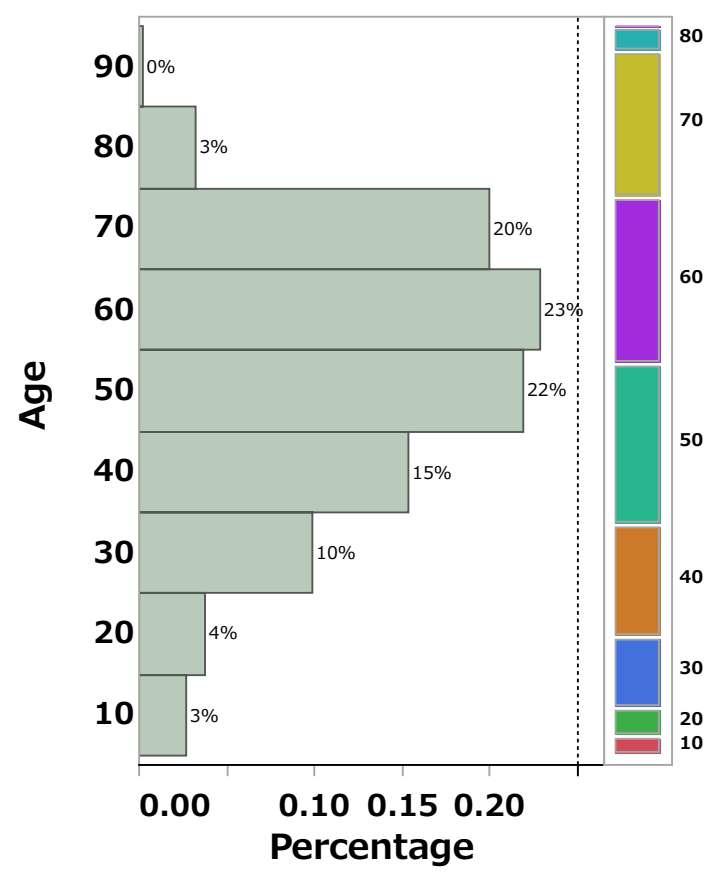

Figure 3. Histogram of age in antibiotic-induced taste and smell disorders. Age was stratified into 10-year groups. The feature of taste and smell disorders peaked in the $60 \mathrm{~s}(23 \%)$, followed by the $50 \mathrm{~s}(22 \%), 70 \mathrm{~s}(20 \%)$, and $40 \mathrm{~s}(15 \%)$.
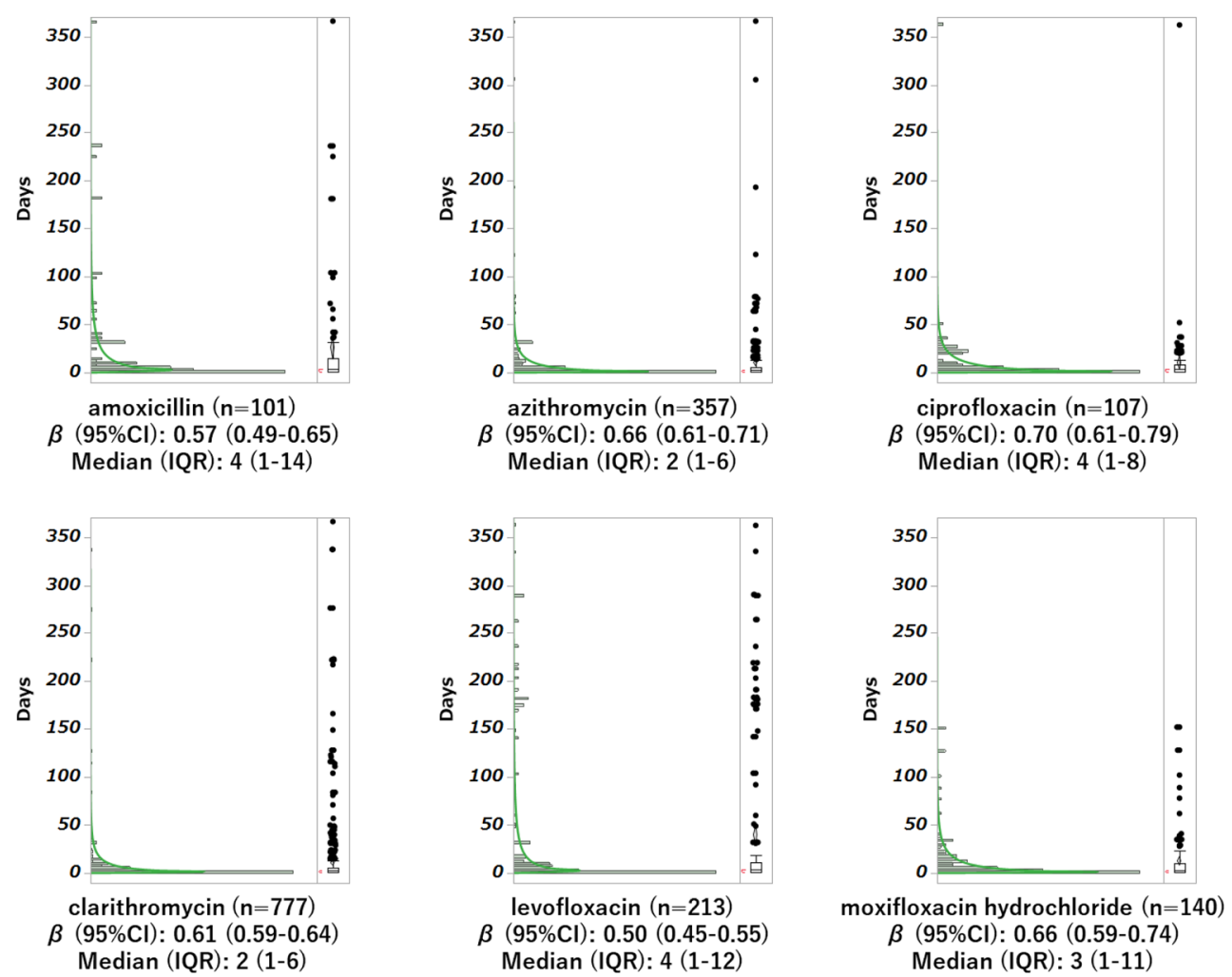

Figure 4. Histogram of antibiotic-induced taste and smell disorders and weibull shape parameters. Adverse event onset time analysis was performed up to 366 days. The median (box center line) and the $25 \%$ and $75 \%$ quartiles (box ends) are shown to the right of each histogram. The whiskers represent the extreme points \pm 1.5 from both ends of the box and represent the largest and smallest values (excluding outliers) of the data within the range. The reliability diamond contains the mean and $95 \%$ confidence interval. Parentheses outside the box indicate the shortest range in which $50 \%$ of the data are clustered. 


\begin{tabular}{|c|c|c|c|c|c|c|c|c|}
\hline \multirow[b]{2}{*}{ Drug } & \multicolumn{2}{|c|}{ Scale parameter } & \multicolumn{2}{|c|}{ Shape parameter } & \multirow[b]{2}{*}{ Median (day) } & \multicolumn{2}{|c|}{$\begin{array}{l}\text { Interquartile } \\
\text { range }\end{array}$} & \multirow[b]{2}{*}{$\mathbf{n}$} \\
\hline & $\alpha$ & $95 \% \mathrm{CI}$ & $\beta$ & 95\%CI & & $25 \%$ & $75 \%$ & \\
\hline Amoxicillin & 36.62 & $(23.0-57.7)$ & 0.42 & $(0.37-0.48)$ & 5 & 2 & 42 & 117 \\
\hline Azithromycin & 7.45 & $(6.06-9.13)$ & 0.54 & $(0.50-0.57)$ & 2 & 1 & 6 & 361 \\
\hline Ciprofloxacin & 9.07 & $(6.47-12.6)$ & 0.6 & $(0.53-0.68)$ & 4 & 1 & 8 & 109 \\
\hline Clarithromycin & 10.23 & $(8.56-12.2)$ & 0.41 & $(0.40-0.43)$ & 2 & 1 & 7 & 810 \\
\hline Levofloxacin & 29.13 & $(20.8-40.6)$ & 0.41 & $(0.38-0.45)$ & 5 & 1 & 32 & 229 \\
\hline Moxifloxacin hydrochloride & 13.24 & $(8.97-19.4)$ & 0.45 & $(0.40-0.50)$ & 4 & 1 & 12 & 145 \\
\hline
\end{tabular}

Table 3. $\beta$ by fitting of weibull distribution. The calculated $\beta$ was below 1 , and the $95 \%$ CI did not include 1 . The occurrence of these antibiotic-induced taste and smell disorders was presumed to be an early failure type that is likely to occur early.

\begin{tabular}{|l|l|l|l|}
\hline & AdOR & 95\% CI & P value \\
\hline Repoting year & 0.99 & $(0.98-1.00)$ & 0.002 \\
\hline Older people $(\geqq 60)$ & 1.17 & $(1.13-1.21)$ & $<.0001$ \\
\hline Gender (man) & 0.88 & $(0.85-0.91)$ & $<.0001$ \\
\hline (Con) hypertension & 1.06 & $(1.00-1.11)$ & 0.043 \\
\hline$($ Con) circulatory disease & 0.59 & $(0.54-0.64)$ & $<.0001$ \\
\hline (Con) mental disorder & 1.09 & $(1.02-1.16)$ & 0.014 \\
\hline (Con) renal disease & 0.35 & $(0.24-0.47)$ & $<.0001$ \\
\hline (Con) cancer & 1.49 & $(1.44-1.55)$ & $<.0001$ \\
\hline Azithromycin & 2.78 & $(1.94-3.84)$ & $<.0001$ \\
\hline clarithromycin & 4.14 & $(3.24-5.20)$ & $<.0001$ \\
\hline Clindamycin hydrochloride & 1.44 & $(0.52-3.13)$ & 0.415 \\
\hline Doxycycline & 4.8 & $(3.00-7.23)$ & $<.0001$ \\
\hline Levofloxacin & 1.67 & $(1.19-2.27)$ & 0.002 \\
\hline Moxifloxacin hydrochloride & 2.4 & $(1.47-3.68)$ & $2 \mathrm{E}-04$ \\
\hline
\end{tabular}

Table 4. AdORs and $P$ values as determined via multiple logistic regression analysis. (Con): concomitant diseases.

\begin{tabular}{|c|c|c|c|c|c|c|c|c|c|c|c|c|}
\hline \multirow[b]{2}{*}{ Drug Name } & \multicolumn{2}{|c|}{ All Date } & \multicolumn{2}{|c|}{$\begin{array}{c}\text { Non- } \\
\text { (Con) Hypertension }\end{array}$} & \multicolumn{2}{|c|}{$\begin{array}{c}\text { Non- } \\
\text { (Con) Circulatory disease }\end{array}$} & \multicolumn{2}{|c|}{$\begin{array}{c}\text { Non- } \\
\text { (Con) Mental disorder }\end{array}$} & \multicolumn{2}{|c|}{$\begin{array}{c}\text { Non- } \\
\text { (Con) Renal disease }\end{array}$} & \multicolumn{2}{|c|}{$\begin{array}{c}\text { Non- } \\
\text { (Con) Cancer }\end{array}$} \\
\hline & $\begin{array}{c}\text { ROR } \\
(95 \% \mathrm{Cl}) \\
\end{array}$ & P-value & $\begin{array}{c}\text { ROR } \\
(95 \% \mathrm{Cl}) \\
\end{array}$ & P-value & $\begin{array}{c}\text { ROR } \\
(95 \% \mathrm{Cl}) \\
\end{array}$ & P-value & $\begin{array}{c}\mathrm{ROR} \\
(95 \% \mathrm{Cl}) \\
\end{array}$ & P-value & $\begin{array}{c}\text { ROR } \\
(95 \% \mathrm{Cl}) \\
\end{array}$ & $\mathrm{P}$-value & $\begin{array}{c}\mathrm{ROR} \\
(95 \% \mathrm{Cl}) \\
\end{array}$ & P-value \\
\hline amoxicillin & $\begin{array}{c}1.09 \\
(0.94-1.26)\end{array}$ & 0.2744 & $\begin{array}{c}0.64 \\
(0.45-0.91)\end{array}$ & 0.008 & $\begin{array}{c}0.70 \\
(0.51-0.97)\end{array}$ & 0.0234 & $\begin{array}{c}0.62 \\
(0.44-0.87)\end{array}$ & 0.0027 & $\begin{array}{c}0.66 \\
(0.48-0.91)\end{array}$ & 0.0064 & $\begin{array}{c}0.65 \\
(0.47-0.91)\end{array}$ & 0.0086 \\
\hline azithromycin & $\begin{array}{c}5.50 \\
(5.15-5.87)\end{array}$ & $<.0001$ & $\begin{array}{c}2.96 \\
(2.46-3.57)\end{array}$ & $<.0001$ & $\begin{array}{c}2.81 \\
(2.34-3.38)\end{array}$ & $<.0001$ & $\begin{array}{c}2.75 \\
(2.29-3.31)\end{array}$ & $<.0001$ & $\begin{array}{c}2.75 \\
(2.75-3.29)\end{array}$ & $<.0001$ & $\begin{array}{c}2.89 \\
(2.41-3.46)\end{array}$ & $<.0001$ \\
\hline ciprofloxacin & $\begin{array}{c}1.04 \\
(0.95-1.15)\end{array}$ & 0.3962 & $\begin{array}{c}0.57 \\
(0.43-0.76)\end{array}$ & $<.0001$ & $\begin{array}{c}0.56 \\
(0.42-0.74)\end{array}$ & $<.0001$ & $\begin{array}{c}0.56 \\
(0.42-0.74)\end{array}$ & $<.0001$ & $\begin{array}{c}0.55 \\
(0.42-0.72)\end{array}$ & $<.0001$ & $\begin{array}{c}0.61 \\
(0.46-0.80)\end{array}$ & 0.0001 \\
\hline clarithromycin & $\begin{array}{c}7.67 \\
(7.22-8.16)\end{array}$ & $<.0001$ & $\begin{array}{c}2.26 \\
(1.87-2.74)\end{array}$ & $<.0001$ & $\begin{array}{c}2.61 \\
(2.19-3.12)\end{array}$ & $<.0001$ & $\begin{array}{c}2.42 \\
(2.03-2.89)\end{array}$ & $<.0001$ & $\begin{array}{c}2.31 \\
(1.94-2.76)\end{array}$ & $<.0001$ & $\begin{array}{c}2.40 \\
(2.01-2.87)\end{array}$ & $<.0001$ \\
\hline clindamycin hydrochloride & $\begin{array}{c}2.15 \\
(1.81-2.54)\end{array}$ & $<.0001$ & $\begin{array}{c}0.76 \\
(0.43-1.36)\end{array}$ & 0.3653 & $\begin{array}{c}0.74 \\
(0.42-1.32)\end{array}$ & 0.3079 & $\begin{array}{c}0.69 \\
(0.39-1.23)\end{array}$ & 0.2169 & $\begin{array}{c}0.68 \\
(0.38-1.21)\end{array}$ & 0.1793 & $\begin{array}{c}0.59 \\
(0.31-1.12)\end{array}$ & 0.0798 \\
\hline doxycycline & $\begin{array}{c}1.48 \\
(1.23-1.78)\end{array}$ & 0.0001 & $\begin{array}{c}3.51 \\
(2.73-4.52)\end{array}$ & $<.0001$ & $\begin{array}{c}3.38 \\
(2.62-4.35)\end{array}$ & $<.0001$ & $\begin{array}{c}3.16 \\
(2.44-4.08)\end{array}$ & $<.0001$ & $\begin{array}{c}3.04 \\
(2.35-3.93)\end{array}$ & $<.0001$ & $\begin{array}{c}3.47 \\
(2.66-4.53)\end{array}$ & $<.0001$ \\
\hline levofloxacin & $\begin{array}{c}1.59 \\
(1.44-1.75)\end{array}$ & $<.0001$ & $\begin{array}{c}1.72 \\
(1.45-2.03)\end{array}$ & $<.0001$ & $\begin{array}{c}1.53 \\
(1.28-1.81)\end{array}$ & $<.0001$ & $\begin{array}{c}1.62 \\
(1.37-1.91)\end{array}$ & $<.0001$ & $\begin{array}{c}1.54 \\
(1.31-1.82)\end{array}$ & $<.0001$ & $\begin{array}{c}1.69 \\
(1.43-2.01)\end{array}$ & $<.0001$ \\
\hline moxifloxacin hydrochloride & $\begin{array}{c}2.84 \\
(2.50-3.22) \\
\end{array}$ & $<.0001$ & $\begin{array}{c}1.92 \\
(1.37-2.69) \\
\end{array}$ & 0.0007 & $\begin{array}{c}2.23 \\
(1.65-3.01) \\
\end{array}$ & $<.0001$ & $\begin{array}{c}1.83 \\
(1.31-2.54) \\
\end{array}$ & 0.0013 & $\begin{array}{c}2.21 \\
(1.65-2.96) \\
\end{array}$ & $<.0001$ & $\begin{array}{c}2.28 \\
(1.70-3.07) \\
\end{array}$ & $<.0001$ \\
\hline
\end{tabular}

Table 5. ROR of antibiotics that cause taste and smell disorders adjusted for the effects of concomitant disease. A signal was determined to be present if the lower limit of the $95 \%$ CI of the recalculated ROR was greater than 1. If a signal was determined to be undetectable after adjustment for confounding factors, the signal intensity of that drug before adjustment was a false signal due to confounding factors. 
that did not result in a false signal, confirming that confounding factors may have resulted in an increased signal intensity estimate. Furthermore, these results were like those of AdOR calculated by logistic regression analysis.

\section{Discussion}

The study detected signals for taste and smell disorders for six antibiotics. However, the possibility of a false signal was considered regarding clindamycin. For some of these drugs, taste and smell disorders is not listed as an $\mathrm{AE}$ in the package insert in Japan. Fluoroquinolone antibiotics are known to inhibit zinc absorption by forming chelates with zinc. In addition, tetracycline antibiotics are also known to inhibit zinc absorption ${ }^{20}$. In addition, it has been reported that macrolide antibiotics decrease the blood levels of divalent cation elements (calcium and magnesium $)^{21}$. Based on the interactions among these metal cations and antibiotics, it is considered that taste cell turnover ${ }^{22}$, which is generally considered to require approximately 10 days, is delayed, resulting in taste and smell disorders. Additionally, drug-induced olfactory disturbances are thought to be caused by damage to the olfactory nerve itself, which is in the olfactory mucosa and can cause olfactory neuropathy. It has been suggested that gender might affect the onset of drug-induced taste and smell disorders ${ }^{23}$. Some reports in Japan revealed that its incidence is higher in women ${ }^{6}$, in line with our findings. Regarding the spontaneous reporting system, it is more frequently reported in women than in men, which may affect the signal intensity. However, based on the results of this study, caution must be exercised when administering the drug to women.

Generally, drug-induced AEs are more likely to occur in older individuals than in younger individuals. Past research reported that the risk of taste and smell disorders increases with aging ${ }^{24}$. This finding is believed to be attributable to nutritional deficiency caused by decreases in appetite and salivary secretion, resulting in decreased zinc absorption and delayed taste cell turnover. Given that antibiotics can inhibit the absorption of zinc ${ }^{25}$, it is possible that these drugs have direct effects on taste buds in the oral cavity.

Conversely, older individuals are more frequently prescribed drugs that reduce salivation, and they are more frequently prescribed multiple drugs simultaneously ${ }^{26}$. In addition, changes in saliva properties with aging can affect taste and taste performance ${ }^{27}$. In addition, age-related loss of olfactory cells and neurodegenerative diseases, such as Alzheimer's disease, can affect the sense of smell and olfactory performance. The increased risk of the development of antibiotic-induced taste and smell disorders in patients over 60 years of age identified in the present study confirms the results of our previous study ${ }^{7}$. This analysis revealed that patients with a history of hypertension, psychiatric disorders and cancer were more likely to develop drug-induced taste and smell disorders. These factors may be associated with treatment with anticholinergic drugs, ARB/ACE inhibitors, and chemotherapy and other anti-cancer treatments. Taste and smell disorders have also been associated with antibiotics marketed for ophthalmic use $(0.5 \%$ of the reports of taste and smell disorders for which the route of administration could be ascertained). Some ophthalmic preparations marketed in Japan have taste and smell disorders listed as side effects in the package inserts, and although the frequency of such cases is considered low, caution is considered necessary for ophthalmic preparations as well. The purpose of the analysis of AE onset time was to compare the risk and developmental profile of taste and smell disorders in the clinic using new information ${ }^{28}$. The present study revealed that the onset of antibiotic-induced taste and smell disorders peaked at 1 week and decreased over time. Based on these findings, we recommend that patients taking fluoroquinolone, macrolides, or tetracycline antibiotics be monitored for at least 1 week and then followed for about 2 months. This study had some limitations. SRSs such as FAERS are affected by over-reporting, under-reporting, missing data, the exclusion of healthy individuals, the lack of denominator, and the presence of confounders. Thus, such studies cannot quantify the true risk. For example, ROR examines only the increased risk of AE reporting but not the risk of AE occurrence. Therefore, care must be taken in interpreting the results from the FAERS database. In the case of taste and smell disorders, there is a possibility of under-reporting due to the nature of subjective symptoms. In addition, no analysis of antibiotic dosage was conducted, so it is not clear what the dosage limit is. Moreover, information on the route of administration, concomitant medications, and antibiotic combination therapy were not analyzed in this study. These factors may affect the development of taste and smell disorders and should be considered confounding factors and should be addressed in future studies. This study analyzed taste and smell disorders by stratifying the cases. On the other hand, since patients may contribute to multiple reports in FAERS, furth Alzheimer insights may be gained by analyzing indications and concomitant medications as covariates. Since these analyses may shed light on the effects of disease and concomitant medications on taste and smell disorders, we are considering conducting further analyses.

\section{Conclusion}

This is the first study to evaluate the association between antibiotics and taste and smell disorders using the FAERS database and to analyze the onset of taste and smell disorders induced by individual antibiotics. Finally, accurate signals of antibiotic-related taste and smell disorders were detected for five drugs. In addition, taste and smell disorders is not listed as an AE in the package inserts of some of the identified drugs. The relationship between patient information and antibiotic-induced taste and smell disorders confirms observations from previous studies regarding gender and age differences. The weibull distribution analysis indicated that the identified antibiotics cause an early type of taste and smell disorders. The results of this study stress the need for careful patient monitoring after antibiotics are prescribed. It should also help minimize the risk of the development of antibiotic-induced taste and smell disorders to develop.

\section{Methods}

Creating a data table. FEARS is a database that uses the large-scale SRS published by the US FDA. We performed this analysis using JAPIC AERS provided by the Japan Pharmaceutical Information Center (JAPIC). Data from November 1997 to March 2017 were included. 


\begin{tabular}{|l|l|}
\hline \multicolumn{2}{|l|}{$\begin{array}{l}\text { Taste and Smell disorders } \\
\text { (SMQ:20000046) }\end{array}$} \\
\hline PT & \\
\hline 10001480 & Ageusia \\
\hline 10002653 & Anosmia \\
\hline 10013911 & Dysgeusia \\
\hline 10064480 & Gustometryabnormal \\
\hline 10019071 & Hallucinationgustatory \\
\hline 10019072 & Hallucination, olfactory \\
\hline 10069147 & Hypergeusia \\
\hline 10020989 & Hypogeusia \\
\hline 10050515 & Hyposmia \\
\hline 10056388 & Olfactorynervedisorder \\
\hline 10062927 & Olfactorytestabnormal \\
\hline 10034018 & Parosmia \\
\hline
\end{tabular}

Table 6. Definition of taste and smell disorders. In the extraction by “Taste and smell disorders (SMQ: 2,000,046)", 12 preferred terms (PTs) were included, including six PTs related to taste and six PTs related to smell.

JAPIC AERS is data cleaned and provided by JAPIC so that it can be collated and analyzed using the FAERS data published by the U.S. FDA, which operates FAERS. Data are only available for contracted customers ${ }^{29}$.

FEARS consist of seven data tables (DRUG), adverse event information (REAC), case information (DEMO), treatment information (THER), underlying disease information (INDI), outcome information, and reporter information. The DRUG, REAC, DEMO, THER and INDI tables were joined using the ID numbers assigned to each of these tables. In the DRUG table, drugs are classified into four categories, first suspected drug (PS), second suspected drug (SS), concomitant drug, and interaction, according to the degree of involvement in the expected AE. In the JAPIC AERS, data entries in the THER table are treated as missing data if they cannot be converted to the eight-digit date type (YYYYMMDD); hence, in the DEMO table and THER table, 4,486,811 of the $8,597,728$ entries $(48 \%)$ and $2,372,833(78 \%)$ of the $10,781,670$ cases were reported in the "YYYYMMDD" format, respectively.

In this study, only reports recorded in PS and SS were used. Antibiotics were extracted from the drugs to be analyzed using systemic antibacterial drugs (J01) based on the WHO Anatomical Therapeutic Chemical Classification System. JAPIC AERS contains JAPIC dictionary files that can be linked to DRUG tables (Medicinal Products-Names of Components, Medicinal Products-ATC Classification [Anatomical Therapeutic Chemistry]). Reports for genders other than male and female have been deleted. Age only used reports recorded in "YR," unknown data or data for patients beyond the age of 130 years were deleted. We also differentiated age into younger individuals and older individuals based on age 60 .

Definition of taste and smell disorders. Diseases in the REAC and INDI tables are based on medical terms as preferred terms (PTs) in the ICH International Medical Dictionary for Regulatory Activities/Japanese (MedDRA/J). The MedDRA Standard Query (SMQ) is a group of MedDRA terms related to a defined medical condition or area of interest.

In this study, "Taste and smell disorders (SMQ: 20,000,046)" described in MedDRA/J ver. 18.0 were analyzed. The SMQ contained 12 PTs (Table 6). These extracted PTs were designated as "taste and smell disorders."

Time to AE onset. In the analysis of the time to onset of AEs, only data for patients who reported taste and smell disorders among those treated with antibiotics were extracted. The number of days required to develop taste and smell disorders was calculated from the administration start date of the suspect drug reported in FAERS and the onset date of taste and smell disorders ${ }^{17,26}$. The weibull shape parameter test is used to analyze time-to-onset data, and it can describe the non-constant rate of AE feature. Unknown reports on the date of treatment initiation or the onset of AEs were deleted. In this study, we analyzed more than 100 reported antibiotics and calculated the number of days until AE onset by subtracting the date of drug initiation from the date of AE onset. One was added to the calculated number of days because the start date of the drug was set as day 1 . The upper limit of time to AE onset was set to 366 days.

Multiple logistic regression analysis. AdORs were calculated by the covert adjustment method using a multiple logistic regression equation to correct for the influence of patient background ${ }^{12,14,15}$. Based on a report by Syed et al. ${ }^{1}$, age, gender, and concomitant disease (hypertension, cardiovascular disease, cancer, mental disorder, renal failure) were used as coverts.

Hypertension, cardiovascular disease, cancer, mental disorder, and renal failure comprised the concomitant disease for multiple logistic regression analysis considering the patient background (Table 7). SMQ and Organ 


\begin{tabular}{|c|c|c|c|c|c|}
\hline \multicolumn{2}{|c|}{ (Con) Hypertension } & \multicolumn{2}{|c|}{ (Con) Circulatory disease } & \multicolumn{2}{|c|}{ (Con) Cancer } \\
\hline SMQ & & SMQ & & SMQ & \\
\hline 20000130 & Pulmonary hypertension & 20000001 & Torsades de pointes/QT prolonged & 20000092 & Malignant disorder-related state \\
\hline \multirow[t]{3}{*}{20000147} & Hypertension & 20000004 & Heart failure & 20000094 & Tumor marker \\
\hline & & 20000047 & Myocardial infarction & 20000110 & Neoplasm of the oropharynx \\
\hline & & 20000051 & $\begin{array}{l}\text { A laboratory study, a sign associated with } \\
\text { arrhythmia and symptom }\end{array}$ & 20000194 & Malignant tumor \\
\hline \multicolumn{2}{|c|}{ (Con) Mental disorder } & 20000055 & Sinus node dysfunction & 20000195 & Tumor unidentified in detail \\
\hline SMQ & & 20000056 & Conduction disorders & 20000196 & Malignant biliary tract neoplasm \\
\hline 20000117 & Psychosis and psychopathic disorder & 20000057 & Supraventricular tachyarrhythmia & 20000197 & Biliary tract neoplasm unknown in detail \\
\hline 20000142 & Hostility/aggressiveness & 20000058 & Ventricular tachyarrhythmia & 20000198 & Malignant breast tumour \\
\hline \multirow[t]{3}{*}{20000167} & Depression (except suicide/self-harm) & 20000067 & $\begin{array}{l}\text { Shock-related circulation or heart state } \\
\text { (except torsades de pointes) }\end{array}$ & 20000199 & Breast tumor unknown in detail \\
\hline & & 20000068 & $\begin{array}{l}\text { Torsades de pointes, arterial embolus that } \\
\text { is in a shock-related state and thrombus }\end{array}$ & 20000200 & Malignant ovarian tumor \\
\hline & & 20000082 & Arterial embolus and thrombus & 20000201 & Ovarian tumor unidentified in detail \\
\hline \multicolumn{2}{|c|}{ (Con) Renal disease } & 20000083 & $\begin{array}{l}\text { Of unknown vascular type or mixed } \\
\text { embolus and thrombus }\end{array}$ & 20000202 & Malignant prostate tumor \\
\hline SMQ & & 20000084 & Venous embolus and thrombus & 20000203 & Prostate tumor unidentified in detail \\
\hline 20000003 & Acute renal failure & 20000150 & Cardiomyopathy & 20000204 & Malignant skin tumor \\
\hline 20000181 & Renal vessel disorder & 20000162 & Nonspecific arrhythmia term & 20000205 & Skin tumor unidentified in detail \\
\hline \multirow[t]{5}{*}{20000213} & Chronic kidney disease & 20000163 & Nonspecific bradyarrhythmia term & 20000206 & Malignant uterus/salpingioma \\
\hline & & 20000164 & Nonspecific tachyarrhythmia term & 20000207 & Uterus/salpingioma unidentified in detail \\
\hline & & 20000168 & Other ischemic heart disease & 20000208 & Malignant hepatophyma \\
\hline & & & & 20000209 & Hepatophyma unidentified in detail \\
\hline & & & & 20000215 & Malignant lymphoma \\
\hline
\end{tabular}

Table 7. Definition of cancer history. Concomitant disease examined hypertension, cardiovascular disease, cancer, mental disorder, and renal failure. Each of these combined multiple SMQs and defined them as one concomitant disease. One group was used for hypertension, three groups for mental disorder, three groups for renal failure, 15 groups for cardiovascular disease, and 20 groups for cancer. (Con): concomitant diseases.

Classification were used to extract concomitant disease data. The estimated models were evaluated by the goodness of fit test.

Calculation of signal intensity adjusting for confounding factors. Reports of concomitant disease that were associated with taste and smell disorders were excluded from the analysis. After removal of concomitant diseases, signal intensity was re-evaluated to assess false positive results. Concomitant diseases were defined as hypertension, cardiovascular disease, cancer, mental disorder, and renal failure. If the signal was determined to be undetectable after adjustment for concomitant diseases, the signal intensity for that drug before adjustment was determined to be a false signal due to confounders.

Assessment of false positives in signal detection. For the history of the current disease, the target disease was selected by multiple logistic regression analysis. A signal was considered present if the lower limit of the $95 \%$ CI of the recalculated ROR was $>1$. If a signal was considered undetectable after adjustment for confounding factors, the signal intensity of that drug before adjustment was deemed to be a false signal due to confounding factors.

Analytical method. Based on the two classifications of taste and smell disorders and suspected drugs, a two-by-two frequency table was prepared, ROR and 95\% CI (95\% CI) were calculated, and Fisher's direct exact test was performed. We compiled a cross-tabulation table based on two classifications: the presence or absence of taste and smell disorders and the presence or absence of the suspected drugs. Therefore, we calculated the $P$ values using Fisher's exact test and RORs (Fig. 5). RORs cannot be calculated if there are cells with 0 in the cross-tabulation table, and the estimation becomes unstable if the frequency is small. To correct for this bias, a correction was made to increase the values in all cells by 0.5 (Haldane-Anscombe correction) ${ }^{30}$.

When the lower limit of the calculated $95 \%$ CI of ROR exceeded 1, it was judged that there was a signal ${ }^{31}$. A scatter plot was created (volcano plot) with the logarithm of ROR as the X-axis and the negative logarithm of $P$ value calculated using Fisher's exact test as the Y-axis to identify drugs associated with taste and smell disorders ${ }^{18,32}$. In this study, we analyzed antibiotics with more than 100 reports. The time to the onset of taste and smell disorders was calculated as the time from the date of treatment initiation to symptom onset. The scale parameter $\alpha$ of the weibull distribution determines the scale of the distribution function. The shape parameter $\beta$ of the weibull distribution indicates the hazard without a reference population. When $\beta$ equals 1 , the hazard 


\begin{tabular}{lcc}
\hline & $\begin{array}{c}\text { Taste and smell } \\
\text { disorders }\end{array}$ & All other Aes \\
\hline $\begin{array}{l}\text { Reports with the } \\
\text { suspected drugs }\end{array}$ & $a$ & $c$ \\
All of reports & $b$ & $d$ \\
\hline $\boldsymbol{R O} R=\frac{\boldsymbol{a} / \boldsymbol{b}}{\boldsymbol{c} / \boldsymbol{d}}=\frac{\boldsymbol{a d}}{\boldsymbol{b} \boldsymbol{c}}$ &
\end{tabular}

Figure 5. Cross-tabulation and calculation formula of for the reporting odds ratio (ROR) of taste and smell disorders. (a) Number of cases of taste and smell disorders attributable to the suspected drug. (b) Number of cases of taste and smell disorders attributable to other drugs. (c) Number of cases of other AEs attributable to the suspected drug. (d) Number of cases of other AEs attributable to other drugs. ROR was calculated using the presented formula.

$$
\log (\text { odds })=\beta_{0}+\beta_{1} Y+\beta_{2} S+\beta_{3} A+\beta_{4} C+\beta_{5} D
$$

( $Y=$ reporting year, $S=$ sex, $A=$ stratified age group, $C=$ concomitant disease, $D=d r u g, \beta_{0-5}=$ model estimate)

Figure 6. Multiple logistic regression equation for calculating the adjusted odds ratio.

is estimated to carry a constant risk over time. When $\beta$ exceeds 1 and the $95 \%$ CI of $\beta$ does not include 1 , the hazard risk is considered to increase over time. When $\beta$ is smaller than 1 and the $95 \% \mathrm{CI}$ of $\beta$ does not include 1 , the hazard risk is considered to decrease over time ${ }^{15,17,18,20,28}$.

Multiple logistic regression analysis was performed to examine the effects of the analyzed drugs and patient background. AdORs were calculated to control for coverts using a multiple logistic regression equation. Patients were stratified by age into younger ( $<60$ years) and older populations $(60-130 \text { years })^{7}$. Gender, age, concomitant disease, and drug use were coded using this stratification (Fig. 6).

The analyzed drugs were determined to be significantly associated with taste and smell disorders based on the ROR signal, and the number of reports was at least 100.

The internal correlation was estimated using the pairwise method. If the square of $\rho$ exceeded 0.9 , then we concluded that an internal correlation existed. In the absence of an internal correlation, we treated these items as independent factors ${ }^{12}$. To detect false signals, we recalculated the signal intensities after removing suspected confounders related to concomitant disease for the antibiotics included in Table 1. Signal intensities for drugs eliciting taste and olfactory disturbances were calculated with and without the removal of the current history of suspected confounding factors. All data were analyzed using JMP Pro 14.3.0 (SAS, Cary, NC).

Received: 24 August 2020; Accepted: 20 April 2021

Published online: 05 May 2021

\section{Reference}

1. Syed, Q., Hendler, K. T. \& Koncilja, K. The impact of aging and medical status on dysgeusia. Am. J. Med. 129, 753. https://doi.org/ 10.1016/j.amjmed.2016.02.003 (2016).

2. Hur, K., Choi, J. S., Zheng, M., Shen, J. \& Wrobel, B. Association of alterations in smell and taste with depression in older adults. Laryngoscope Investig. Otolaryngol. 3, 94-99. https://doi.org/10.1002/lio2.142 (2018).

3. Liu, G., Zong, G., Doty, R. L. \& Sun, Q. Prevalence and risk factors of taste and smell impairment in a nationwide representative sample of the US population: a cross-sectional study. BMJ Open 6, e013246. https://doi.org/10.1136/bmjopen-2016-013246 (2016).

4. Mortazavi, H., Shafiei, S., Sadr, S. \& Safiaghdam, H. Drug-related dysgeusia: A systematic review. Oral Health Prev. Dent. 16, 499-507. https://doi.org/10.3290/j.ohpd.a41655 (2018).

5. Tomomi, T., Masayuki, U., Tomomi, M., Tomoko, N. \& Masafumi, S. Basics and clinics of taste disturbance. Oral. 30, 31-35. https:// doi.org/10.14821/stomatopharyngology.30.31 (2017).

6. Akiko, S. et al. Clinical analysis of 1059 patients with taste disorders. Nippon Jibiinkoka Gakkai Kaiho 116, 77-82. https://doi.org/ 10.3950/jibiinkoka.116.77 (2013).

7. Pugnaloni, S. et al. Modifications of taste sensitivity in cancer patients: a method for the evaluations of dysgeusia. Support Care Cancer 28, 1173-1181. https://doi.org/10.1007/s00520-019-04930-x (2020).

8. Tuccori, M. et al. Drug-induced taste and smell alterations. Drug Saf. 34, 849-859. https://doi.org/10.2165/11593120-00000000000000 (2011).

9. Nakamura, M. et al. Analysis of the time-to-onset of osteonecrosis of jaw with bisphosphonate treatment using the data from a spontaneous reporting system of adverse drug events. J. Pharm. Health Care Sci. 1, 34. https://doi.org/10.1186/s40780-015-0035-2 (2015).

10. Abe, J. et al. Analysis of Stevens-Johnson syndrome and toxic epidermal necrolysis using the Japanese Adverse Drug Event Report database. J. Pharm. Health Care Sci. 2, 14. https://doi.org/10.1186/s40780-016-0048-5 (2016).

11. Hosoya, R., Uesawa, Y., Ishii-Nozawa, R. \& Kagaya, H. Analysis of factors associated with hiccups based on the Japanese Adverse Drug Event Report database. PLoS ONE 12, e0172057. https://doi.org/10.1371/journal.pone.0172057 (2017).

12. Ohyama, K. \& Sugiura, M. Evaluation of the association between topical prostaglandin F2 $\alpha$ analogs and asthma using the JADER database: comparison with $\beta$-blockers. Yakugaku Zasshi 138, 559-564. https://doi.org/10.1248/yakushi.17-00162 (2018). 
13. Suzuki, Y. et al. Analysis of the interaction between clopidogrel, aspirin, and proton pump inhibitors using the FDA adverse event reporting system database. Biol. Pharm. Bull. 38, 680-686. https://doi.org/10.1248/bpb.b14-00191 (2015).

14. Matsui, T. et al. Age-related trends in injection site reaction incidence induced by the tumor necrosis factor- $\alpha$ (TNF- $\alpha$ ) inhibitors etanercept and adalimumab: The food and drug administration adverse event reporting system, 2004-2015. Int. J. Med. Sci. 14, 102-109. https://doi.org/10.7150/ijms.17025 (2017).

15. Hatahira, H. et al. Drug-induced gingival hyperplasia: A retrospective study using spontaneous reporting system databases. J. Pharm. Health Care Sci. 3, 19. https://doi.org/10.1186/s40780-017-0088-5 (2017).

16. Sasaoka, S. et al. Evaluation of the association of hand-foot syndrome with anticancer drugs using the US Food and Drug Administration adverse event reporting system (FAERS) and Japanese adverse drug event report (JADER) databases. Yakugaku Zasshi 136, 507-515. https://doi.org/10.1248/yakushi.15-00222 (2016).

17. Nakao, S. et al. Evaluation of drug-induced photosensitivity using the Japanese adverse drug event report (JADER) database. Biol. Pharm. Bull. 40, 2158-2165. https://doi.org/10.1248/bpb.b17-00561 (2017).

18. Sugawara, H. et al. Analyses of respiratory depression associated with opioids in cancer patients based on the Japanese adverse drug event report database. Biol. Pharm. Bull. 42, 1185-1191. https://doi.org/10.1248/bpb.b19-00105 (2019).

19. Neha, R. et al. Aromatase inhibitors associated osteonecrosis of jaw: Signal refining to identify pseudo safety signals. Int. J. Clin. Pharm. 42, 721-727. https://doi.org/10.1007/s11096-020-01018-z (2020).

20. Mitsuru, K. et al. Drug therapy and oral disorders. Folia Pharmacol. Jpn. 127, 447-453. https://doi.org/10.1254/fpj.127.447 (2006).

21. Foulds, G., Hilligoss, D. M., Henry, E. B. \& Gerber, N. The effects of an antacid or cimetidine on the serum concentrations of azithromycin. J. Clin. Pharmacol. 31, 164. https://doi.org/10.1002/j.1552-4604.1991.tb03701.x (1991).

22. Hidemi, M., Tomomi, R., Tomoko, N., Masanori, U. \& Masafumi, S. Treatment of taste disorder. Oral Med. 31, 149-154. https:// doi.org/10.14821/stomatopharyngology.31.149 (2018).

23. Doty, R. L., Shah, M. \& Bromley, S. M. Drug-induced taste disorders. Drug Saf. 31, 199-215. https://doi.org/10.2165/00002018200831030-00002 (2008)

24. Ikeda, M., Ikui, A., Komiyama, A., Kobayashi, D. \& Tanaka, M. Causative factors of dysgeusia in the elderly, and therapeutic effects of zinc. J. Laryngol. Otol. 122, 155-160. https://doi.org/10.1017/S0022215107008833 (2008).

25. Kouji, A., Toshiro, O., Yuki, Y., Keiko, M. \& Kyoko, I. Postmarketing surveillance of azithromycin in adult patients. Jpn. J. Chemother. 53, 421-430. https://doi.org/10.11250/chemotherapy1995.53.421 (2005).

26. Sayaka, A. et al. Multidrug use positively correlates with high-risk prescriptions in the Japanese elderly: a longitudinal study. J. Pharm. Health Care Sci. 5, 20. https://doi.org/10.1186/s40780-019-0150-6 (2019).

27. Pushpass, R. G., Daly, B., Kelly, C., Proctor, G. \& Carpenter, G. H. Altered salivary flow, protein composition, and rheology following taste and TRP stimulation in older adults. Front. Physiol. 10, 652. https://doi.org/10.3389/fphys.2019.00652 (2019).

28. Masayuki, Y. \& Jun, H. Comparison of onset profiles of various interferon preparations for suicide or diabetes-related side effects. Pharmacoepidemiology 19, 23-30. https://doi.org/10.3820/jjpe.19.23 (2014).

29. Masayuki, H. et al. Factors affecting the timing of signal detection of adverse drug reactions. PLoS ONE 10, e0144263. https://doi. org/10.1371/journal.pone.0144263 (2015).

30. Hiroyuki, W. et al. Early detection of important safety information. Recent methods for signal detection. Jpn. J. Biomet. 25, 37-60. https://doi.org/10.5691/jjb.25.37 (2004).

31. Toshiharu, F. Signal detection of adverse drug reactions. Jpn. J. Pharmacoepidemiol. 14, 27-36. https://doi.org/10.3820/jjpe.14.27 (2009).

32. Junko, N., Yoshihiro, U. \& Hajime, K. Analysis of the tendency of oxycodone to develop side effects based on the database of side effects. Palliative Care Res. 10, 161-168. https://doi.org/10.2512/jspm.10.161 (2015).

\section{Acknowledgements}

We would like to thank Japan Pharmaceutical Information Center (JAPIC) for allowing the use of the JAPIC AERS database under a collaborative research contract.

\section{Author contributions}

Y.K. analyzed the data and created the manuscript. Y.U., J.N. contributed to the concept and design of the study. All authors commented on the manuscript and read and approved the final manuscript.

\section{Competing interests}

The authors declare no competing interests.

\section{Additional information}

Supplementary Information The online version contains supplementary material available at https://doi.org/ 10.1038/s41598-021-88958-2.

Correspondence and requests for materials should be addressed to Y.U.

Reprints and permissions information is available at www.nature.com/reprints.

Publisher's note Springer Nature remains neutral with regard to jurisdictional claims in published maps and institutional affiliations.

(c) (i) Open Access This article is licensed under a Creative Commons Attribution 4.0 International License, which permits use, sharing, adaptation, distribution and reproduction in any medium or format, as long as you give appropriate credit to the original author(s) and the source, provide a link to the Creative Commons licence, and indicate if changes were made. The images or other third party material in this article are included in the article's Creative Commons licence, unless indicated otherwise in a credit line to the material. If material is not included in the article's Creative Commons licence and your intended use is not permitted by statutory regulation or exceeds the permitted use, you will need to obtain permission directly from the copyright holder. To view a copy of this licence, visit http://creativecommons.org/licenses/by/4.0/.

(C) The Author(s) 2021 